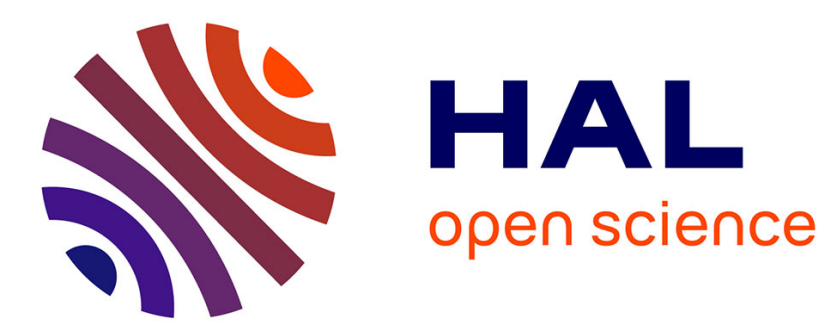

\title{
A new finite element formulation for internal acoustic problems with dissipative walls
}

\author{
Walid Larbi, Jean-François Deü, Roger Ohayon
}

\section{To cite this version:}

Walid Larbi, Jean-François Deü, Roger Ohayon. A new finite element formulation for internal acoustic problems with dissipative walls. International Journal for Numerical Methods in Engineering, 2006, 68, pp.381 - 399. 10.1002/nme.1727 . hal-01422437

\section{HAL Id: hal-01422437 \\ https://hal.science/hal-01422437}

Submitted on 25 Dec 2016

HAL is a multi-disciplinary open access archive for the deposit and dissemination of scientific research documents, whether they are published or not. The documents may come from teaching and research institutions in France or abroad, or from public or private research centers.
L'archive ouverte pluridisciplinaire HAL, est destinée au dépôt et à la diffusion de documents scientifiques de niveau recherche, publiés ou non, émanant des établissements d'enseignement et de recherche français ou étrangers, des laboratoires publics ou privés. 


\title{
A new finite element formulation for internal acoustic problems with dissipative walls
}

\author{
W. Larbi, J.-F. Deü and R. Ohayon \\ Structural Mechanics and Coupled Systems Laboratory, Conservatoire National des Arts et Métiers, \\ 292 rue Saint-Martin, 75141 Paris Cedex 03, France
}

This work concerns the variational formulation and the numerical computation of internal acoustic problems with absorbing walls. The originality of the proposed approach, compared to other existing methods, is the introduction of the normal fluid displacement field on the damped walls. This additional variable allows to transpose formulations in frequency domain to time domain when the fluid is described by a scalar field (pressure or fluid displacement potential). With this new scalar unknown, various absorbing wall models can be introduced in the variational formulation. Moreover, the associated finite element matrix system in symmetric form can be solved in frequency and time domain.

KEY WORDS: dissipative acoustics; noise reduction; finite element method; symmetric formulation; wall normal fluid displacement field

\section{INTRODUCTION}

In this paper, we are interested in the linear oscillations of an acoustic (i.e. inviscid, compressible, barotropic) fluid contained in a rigid cavity, with some or all of its walls covered by a thin layer of absorbing material able to dissipate part of the acoustic energy of the fluid.

Recently, considerable research has been devoted to the development and testing of noise reduction techniques by passive damping treatments. Many approaches have been proposed. For instance, porous materials have been widely used for noise and vibration control purposes. For example, a study of flexible porous damping materials is presented by Göransson [1], where a finite element solution is proposed for the coupled acoustic-elastic wave propagation problem. 
In a related approach, a wall impedance, characterizing the absorptive material, is used to take into account the effect of damping material. Let us mention the papers [2-4] where a wall impedance is introduced to describe the absorbing boundary conditions at dissipative walls. It can be noted that for a given material, the parameters of this impedance can be found experimentally [5].

Different fields can be chosen to describe the behaviour of the fluid: pressure, fluid displacement, fluid potential displacement or fluid potential velocity (see, for example, [6-8]). In the particular case of dissipative acoustic problem, a pressure/displacement potential formulation in frequency domain of the fluid is given and numerically solved by finite element and modal reduction methods [2]. It should be noted that in this case, the frequency complex dependence does not permit to write the associated problem in time domain. In Reference [3], a displacement formulation of dissipative acoustic problem is proposed. In this case, one has to pay attention to the discretization of the admissible class of irrotational motions. Special complex treatments are then required (Raviard-Thomas types of finite elements) and this vector formulation can lead to costly computation.

The aim of this paper is to develop a finite element formulation for damped acoustic cavity, which is valid in time and frequency domains using acoustic pressure as main variable. In order to achieve this objective, a new scalar variable is introduced to describe the damping interface namely the normal fluid displacement field. With this new scalar unknown, various interface damping models can be taken into account in the variational formulation. Moreover, the associated finite element matrix system is linear and can be solved in frequency and time domains. This new formulation leads to an unsymmetric matrix equation. A symmetric formulation can be obtained through the introduction of an intermediate unknown field, namely displacement potential of the fluid [6]. Numerical examples are presented in order (i) to validate the new approach through comparison with modal results given in literature, and (ii) to evaluate the influence of the damping model on the dynamic response of the system. In this last case, a modal reduction approach is used in conjunction with a direct time integration method.

\section{DISSIPATIVE ACOUSTIC BASIC LOCAL EQUATIONS}

Consider an inviscid compressible barotropic fluid contained in a rigid cavity, with some of its walls covered by a thin layer of a viscoelastic material, in order to absorb part of the acoustic energy of the fluid. Let us denote by $\Omega_{\mathrm{F}}$ the domain occupied by the fluid. The boundary of $\Omega_{\mathrm{F}}$ is noted $\partial \Omega_{\mathrm{F}}=\Gamma_{\mathrm{A}} \cup \Gamma_{\mathrm{R}} \cup \Gamma_{\mathrm{P}}$, with $\Gamma_{\mathrm{A}}$ the face covered by damping material and called 'absorbing boundary', $\Gamma_{\mathrm{R}}$ the rigid face and $\Gamma_{\mathrm{P}}$ the face where a given pressure $p^{d}$ is applied. The unit outer normal vector along $\partial \Omega_{\mathrm{F}}$ is denoted by $\mathbf{n}$. Additionally, the following notations for the quantities associated with the fluid: $p$ for the pressure, $\mathbf{u}^{\mathrm{F}}$ for the fluid displacement field, $\rho_{\mathrm{F}}$ for the mass density and $c$ for the speed of sound.

The local equations of the problem described in Figure 1 are

$$
\begin{aligned}
\rho_{\mathrm{F}} \frac{\partial^{2} \mathbf{u}^{\mathrm{F}}}{\partial t^{2}}+\nabla p & =\mathbf{0} \quad \text { in } \Omega_{\mathrm{F}} \\
p & =-\rho_{\mathrm{F}} c^{2} \operatorname{div} \mathbf{u}^{\mathrm{F}} \quad \text { in } \Omega_{\mathrm{F}}
\end{aligned}
$$




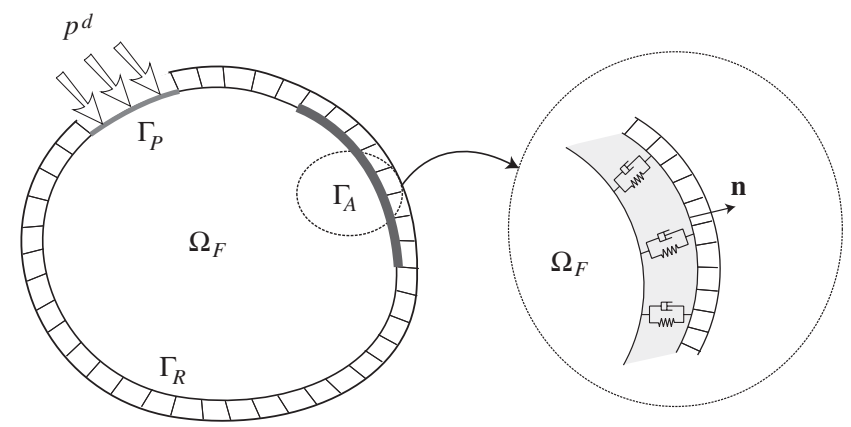

Figure 1. Dissipative acoustic cavity.

$$
\begin{aligned}
\mathbf{u}^{\mathrm{F}} \cdot \mathbf{n} & =0 \quad \text { on } \Gamma_{\mathrm{R}} \\
p & =k^{\mathrm{I}} \mathbf{u}^{\mathrm{F}} \cdot \mathbf{n}+d^{\mathrm{I}} \frac{\partial \mathbf{u}^{\mathrm{F}}}{\partial t} \cdot \mathbf{n} \quad \text { on } \Gamma_{\mathrm{A}} \\
p & =p^{d} \quad \text { on } \Gamma_{P}
\end{aligned}
$$

Equation (1a) corresponds to the linearized Euler equations and Equation (1b) represents the constitutive equation of the barotropic fluid. The usual kinematic interface condition for rigid walls, Equation (1c), has been relaxed in the absorbing boundary to take into account the effect of the viscoelastic material represented by Equation (1d). On the right-hand side of this equation, the first term is proportional to the normal component of displacements and accounts for the elastic behaviour of the material, whereas the second one is proportional to the normal velocity and models the viscous damping. Finally, Equation (1e) represents the pressure force applied to the wall $\Gamma_{P}$ of the cavity.

Starting from these equations, it is possible to obtain a displacement formulation by eliminating $p$ in the previous system. The corresponding admissible space must verify $\operatorname{curl} \mathbf{u}^{\mathrm{F}}=\mathbf{0}$ in $\Omega_{\mathrm{F}}$ in order to avoid, in the discretized case, the possible presence of spurious rotational eigenmodes. We refer to Reference [9] for a deep mathematical and numerical analysis of such formulation (throught the use of Raviart-Thomas finite elements).

\section{DISSIPATIVE ACOUSTIC PROBLEM IN TERMS OF FLUID PRESSURE}

In order to remove the problem of nonphysical modes and to arrive at a more compact system of equations, the acoustic problem is usually described either in terms of pressure or fluid potential displacement [6]. However, in the case of dissipative acoustic cavity, it is not trivial to express the problem in time domain using only these variables. On the other hand, in frequency domain, and without considering the forcing terms (free motion), the problem can be written only in terms of pressure variable by elimination of $\mathbf{u}^{\mathrm{F}}$ in Equations (1) but leads to a nonlinear eigenvalue problem as described below. In this case, the pressure is expressed 
in the form $p(x, t)=\mathrm{e}^{\lambda t} p(x)$ and the eigenvalue equations are given by

$$
\begin{aligned}
\Delta p-\frac{\lambda^{2}}{c^{2}} p & =0 \quad \text { in } \Omega_{\mathrm{F}} \\
\nabla p \cdot \mathbf{n} & =0 \quad \text { on } \Gamma_{\mathrm{R}} \\
\nabla p \cdot \mathbf{n} & =\frac{-\rho_{\mathrm{F}} \lambda^{2}}{k^{\mathrm{I}}+\lambda d^{\mathrm{I}}} p \quad \text { on } \Gamma_{\mathrm{A}} \\
p & =0 \quad \text { on } \Gamma_{P}
\end{aligned}
$$

Equation (2a) corresponds to Helmholtz equation in frequency domain (considering $\lambda=-\mathrm{i} \omega$ ) deduced by the elimination of $\mathbf{u}^{\mathrm{F}}$ between Equations (1a) and (1b); Equation (2b) represents the rigid boundary condition on $\Gamma_{\mathrm{R}}$; and Equation (2c) represents the absorbing boundary condition on $\Gamma_{\mathrm{A}}$ written in terms of pressure field.

Thus, the variational formulation consists in finding $\lambda$ and $p$ belonging to the admissible space $C_{p}^{\star}=\left\{p \mid p=0\right.$ on $\left.\Gamma_{p}\right\}$, such that $\forall \delta p \in C_{p}^{\star}$ :

$$
\int_{\Omega_{\mathrm{F}}} \nabla p \cdot \nabla \delta p \mathrm{~d} x+\rho_{\mathrm{F}} \frac{\lambda^{2}}{k^{\mathrm{I}}+\lambda d^{\mathrm{I}}} \int_{\Gamma_{\mathrm{A}}} p \delta p \mathrm{~d} x+\frac{\lambda^{2}}{c^{2}} \int_{\Omega_{\mathrm{F}}} p \delta p \mathrm{~d} \sigma=0
$$

This formulation corresponds to that one proposed by Kehr-Candille and Ohayon [2], written here in terms of pressure instead of fluid displacement potential in Reference [2]. In the abovementioned paper, the equation is written in the form:

$$
\int_{\Omega_{\mathrm{F}}} \nabla p \cdot \nabla \delta p \mathrm{~d} x-\mathrm{i} \rho_{\mathrm{F}} \frac{\omega}{Z(\omega)} \int_{\Gamma_{\mathrm{A}}} p \delta p \mathrm{~d} x-\frac{\omega^{2}}{c^{2}} \int_{\Omega_{\mathrm{F}}} p \delta p \mathrm{~d} \sigma=0
$$

where $\omega$ is the angular frequency and $Z(\omega)$ is the specific impedance of the absorbing wall, which is related to the damping parameters. For the Kelvin-Voigt model used in this work, and considering $\lambda=-i \omega$, the specific impedance $Z$ of the viscoelastic material is defined in terms of the coefficients $k^{\mathrm{I}}$ and $d^{\mathrm{I}}$ in the previous equation through the expression:

$$
Z(\omega)=d^{\mathrm{I}}+\mathrm{i} \frac{k^{\mathrm{I}}}{\omega}
$$

The real and imaginary parts of this specific impedance can be experimentally measured for any given frequency $\omega$. These quantities are the specific resistance and reactance, respectively. It can be noted that in literature, where the time dependence of oscillating quantities is described by $\mathrm{e}^{\mathrm{i} \omega}$, the reactance is negative. For more details, the reader can refer to Reference [5].

Equation (4) shows that the dissipative acoustic problem written in terms of pressure leads to a nonlinear (nor even quadratic) eigenvalue problem and needs specific algorithms for its resolution. Frequency response functions can be obtained by sweeping frequency. 


\section{DISSIPATIVE ACOUSTIC FORMULATION IN TERMS OF FLUID PRESSURE AND FLUID NORMAL DISPLACEMENT AT THE ABSORBING BOUNDARY}

It has been shown, in the previous section, that the dissipative acoustic problem can be expressed in terms of pressure unknown field in frequency domain and for free motion. In this section, we propose a new formulation, valid for the time and frequency domains, in terms of fluid pressure and fluid normal displacement at the absorbing boundary. In the following, this variable will be noted $\eta=\mathbf{u}^{\mathrm{F}} \cdot \mathbf{n}$. Thus, the dynamic equations are given in terms of this new variable $\eta$ and pressure $p$ :

$$
\begin{aligned}
\Delta p-\frac{1}{c^{2}} \frac{\partial^{2} p}{\partial t^{2}} & =0 \quad \text { in } \Omega_{\mathrm{F}} \\
\nabla p \cdot \mathbf{n} & =0 \quad \text { on } \Gamma_{\mathrm{R}} \\
\nabla p \cdot \mathbf{n} & =-\rho_{\mathrm{F}} \frac{\partial^{2} \eta}{\partial t^{2}} \quad \text { on } \Gamma_{\mathrm{A}} \\
p & =k^{\mathrm{I}} \eta+d^{\mathrm{I}} \frac{\partial \eta}{\partial t} \quad \text { on } \Gamma_{\mathrm{A}} \\
p & =p^{d} \quad \text { on } \Gamma_{P}
\end{aligned}
$$

Equation (6a) corresponds to Helmholtz equation in time domain; Equation (6b) represents the rigid boundary condition on $\Gamma_{\mathrm{R}}$; Equation (6c) represents the absorbing boundary condition on $\Gamma_{\mathrm{A}}$; and Equation (6d) corresponds to the relation between fluid pressure and normal fluid displacement on $\Gamma_{\mathrm{A}}$ deduced from the behaviour of the viscolelastic material.

Multiplying Equation (6a) by a time-independent test function $\delta p \in C_{p}^{\star}$, applying a Green's formula and dividing by $\rho_{\mathrm{F}}$, we have

$$
\frac{1}{\rho_{\mathrm{F}}} \int_{\Omega_{\mathrm{F}}} \nabla p \cdot \nabla \delta p \mathrm{~d} x+\frac{1}{\rho_{\mathrm{F}} c^{2}} \int_{\Omega_{\mathrm{F}}} \frac{\partial^{2} p}{\partial t^{2}} \delta p \mathrm{~d} x+\int_{\Gamma_{\mathrm{A}}} \frac{\partial^{2} \eta}{\partial t^{2}} \delta p \mathrm{~d} \sigma=0
$$

Similarly, let $\delta \eta$ be the time-independent test function, associated to $\eta$, belonging to the admissible space $C_{\eta}$. Multiplying Equation (6d) by $\delta \eta \in C_{\eta}$, we have

$$
\int_{\Gamma_{\mathrm{A}}} p \delta \eta \mathrm{d} \sigma-k^{\mathrm{I}} \int_{\Gamma_{\mathrm{A}}} \eta \delta \eta \mathrm{d} \sigma-d^{\mathrm{I}} \int_{\Gamma_{\mathrm{A}}} \frac{\partial \eta}{\partial t} \delta \eta \mathrm{d} \sigma=0
$$

Thus, the variational (nonsymmetric) formulation of the acoustic cavity problem with absorbing wall consists, for given appropriate initial conditions, in finding $p$ (with $p=p^{d}$ on $\left.\Gamma_{P}\right)$ and $\eta$ such that Equations (7) and (8) are satisfied.

After discretizing by the finite element method, the bilinear forms of the variational formulation involved in Equations (7) and (8), we obtain the following matrix equation:

$$
\left(\begin{array}{cc}
\mathbf{0} & \mathbf{0} \\
\mathbf{C}_{\eta p}^{\mathrm{T}} & \mathbf{K}_{p}
\end{array}\right)\left(\begin{array}{c}
\ddot{\mathbf{H}} \\
\ddot{\mathbf{P}}
\end{array}\right)+\left(\begin{array}{cc}
d^{\mathrm{I}} \mathbf{G}_{\eta} & \mathbf{0} \\
\mathbf{0} & \mathbf{0}
\end{array}\right)\left(\begin{array}{c}
\dot{\mathbf{H}} \\
\dot{\mathbf{P}}
\end{array}\right)+\left(\begin{array}{cc}
k^{\mathrm{I}} \mathbf{G}_{\eta} & -\mathbf{C}_{\eta p} \\
\mathbf{0} & \mathbf{F}_{p}
\end{array}\right)\left(\begin{array}{l}
\mathbf{H} \\
\mathbf{P}
\end{array}\right)=\left(\begin{array}{l}
\mathbf{0} \\
\mathbf{0}
\end{array}\right)
$$


where $\mathbf{H}$ and $\mathbf{P}$ are the vectors of nodal values of $\eta$ and $p$, respectively (a subvector of $\mathbf{P}$ being given on $\Gamma_{P}$ ), and the submatrices of Equation (9) are defined by

$$
\begin{aligned}
\frac{1}{\rho_{\mathrm{F}}} \int_{\Omega_{\mathrm{F}}} \nabla p \cdot \nabla \delta p \mathrm{~d} x & \Rightarrow \delta \mathbf{P}^{\mathrm{T}} \mathbf{F}_{p} \mathbf{P} \\
\frac{1}{\rho_{\mathrm{F}} c^{2}} \int_{\Omega_{\mathrm{F}}} \frac{\partial^{2} p}{\partial t^{2}} \delta p \mathrm{~d} x & \Rightarrow \delta \mathbf{P}^{\mathrm{T}} \mathbf{K}_{p} \ddot{\mathbf{P}} \\
\int_{\Gamma_{\mathrm{A}}} \frac{\partial^{2} \eta}{\partial t^{2}} \delta p \mathrm{~d} \sigma & \Rightarrow \delta \mathbf{P}^{\mathrm{T}} \mathbf{C}_{\eta p}^{\mathrm{T}} \ddot{\mathbf{H}} \\
\int_{\Gamma_{\mathrm{A}}} p \delta \eta \mathrm{d} \sigma & \Rightarrow \delta \mathbf{H}^{\mathrm{T}} \mathbf{C}_{\eta p} \mathbf{P} \\
\int_{\Gamma_{\mathrm{A}}} \eta \delta \eta \mathrm{d} \sigma & \Rightarrow \delta \mathbf{H}^{\mathrm{T}} \mathbf{G}_{\eta} \mathbf{H}
\end{aligned}
$$

The damped vibration modes of the fluid are complex solutions of Equations (7) and (8), of the form $p(x, t)=p(x) \mathrm{e}^{\lambda t}$ and $\eta(x, t)=\eta(x) \mathrm{e}^{\lambda t}$ and setting $p^{d}=0$ on $\Gamma_{P}$. The corresponding matrix quadratic eigenvalue equation is deduced from Equation (9) with $\mathbf{P}=0$ on $\Gamma_{P}$ (for sake of brevity, we have not changed the matrix notations):

$$
\left[\left(\begin{array}{cc}
k^{\mathrm{I}} \mathbf{G}_{\eta} & -\mathbf{C}_{\eta p} \\
\mathbf{0} & \mathbf{F}_{p}
\end{array}\right)+\lambda\left(\begin{array}{cc}
d^{\mathrm{I}} \mathbf{G}_{\eta} & \mathbf{0} \\
\mathbf{0} & \mathbf{0}
\end{array}\right)+\lambda^{2}\left(\begin{array}{cc}
\mathbf{0} & \mathbf{0} \\
\mathbf{C}_{\eta p}^{\mathrm{T}} & \mathbf{K}_{p}
\end{array}\right)\right]\left(\begin{array}{l}
\mathbf{H} \\
\mathbf{P}
\end{array}\right)=\left(\begin{array}{l}
\mathbf{0} \\
\mathbf{0}
\end{array}\right)
$$

We can note that the fluid normal displacement at the absorbing boundary can be expressed in terms of the fluid pressure from the first line of Equation (11):

$$
\mathbf{H}=\frac{1}{k^{\mathrm{I}}+\lambda d^{\mathrm{I}}} \mathbf{G}_{\eta}^{-1} \mathbf{C}_{\eta p} \mathbf{P}
$$

Replacing $\mathbf{H}$ by its expression (12) in the second line of Equation (11), we obtain the following nonlinear eigenvalue problem in terms of $\mathbf{P}$ :

$$
\left(\mathbf{F}_{p}+\frac{\lambda^{2}}{k^{\mathrm{I}}+\lambda d^{\mathrm{I}}} \mathbf{C}_{\eta p}^{\mathrm{T}} \mathbf{G}_{\eta}^{-1} \mathbf{C}_{\eta p}+\lambda^{2} \mathbf{K}_{p}\right) \mathbf{P}=\mathbf{0}
$$

Equation (13) can be directly obtained through discretizing the pressure formulation given in Equation (3). Moreover, it can be easily seen that if $d^{\mathrm{I}}=0$ and $k^{\mathrm{I}}$ tends to infinity, eigenvalues of acoustic problem in rigid cavity are obtained. 


\section{DISSIPATIVE ACOUSTIC SYMMETRIZED FORMULATION IN TERMS OF $(\eta, p)$}

In the previous section, a nonsymmetric quadratic eigenvalue formulation has been obtained. The aim of this section is to establish a symmetric formulation of this dissipative acoustic problem in view of a direct treatment by finite elements. This symmetrization is obtained through introduction of an intermediate unknown field [6]: a fluid displacement potential field $\varphi$, defined up to an additive constant, such that $\mathbf{u}^{\mathrm{F}}=\nabla \varphi$. We shall see in the next section that this problem can be reduced, by means of an elimination procedure, to an eigenvalue problem in terms of $(\eta, p)$.

The equations of the acoustic spectral problem in terms of $(\eta, p, \varphi)$ can be rewitten as follows:

$$
\begin{aligned}
\rho_{\mathrm{F}} \Delta \varphi+\frac{p}{c^{2}} & =0 \quad \text { in } \Omega_{\mathrm{F}} \\
\nabla \varphi \cdot \mathbf{n} & =0 \quad \text { on } \Gamma_{\mathrm{R}} \\
\nabla \varphi \cdot \mathbf{n} & =\eta \quad \text { on } \Gamma_{\mathrm{A}} \\
\varphi & =0 \quad \text { on } \Gamma_{\mathrm{P}} \\
\frac{p}{\rho_{\mathrm{F}} c^{2}} & =-\frac{\lambda^{2}}{c^{2}} \varphi \quad \text { in } \Omega_{\mathrm{F}} \\
-\lambda^{2} \rho_{\mathrm{F}} \varphi & =k^{\mathrm{I}} \eta+\lambda d^{\mathrm{I}} \eta \quad \text { on } \Gamma_{\mathrm{A}}
\end{aligned}
$$

The corresponding variational formulation, involving fluid normal displacement at the absorbing boundary, fluid potential displacement field, and pressure can be easily obtained as described below:

- Let $\delta \varphi$ be the test function, associated to $\varphi$, belonging to the admissible space $C_{\varphi}^{\star}=$ $\left\{\varphi \mid \varphi=0\right.$ on $\left.\Gamma_{p}\right\}$. Multiplying Equation (14a) by $\delta \varphi \in C_{\varphi}^{\star}$ and taking into account Equations (14c) and (14b), we obtain

$$
-\rho_{\mathrm{F}} \int_{\Omega_{\mathrm{F}}} \nabla \varphi \cdot \nabla \delta \varphi x+\rho_{\mathrm{F}} \int_{\Gamma_{\mathrm{A}}} \eta \delta \varphi \mathrm{d} \sigma+\frac{1}{c^{2}} \int_{\Omega_{\mathrm{F}}} p \delta \varphi \mathrm{d} x=0 \quad \forall \delta \varphi \in C_{\varphi}^{\star}
$$

- Multiplying Equation (14e) by a test function $\delta p \in C_{p}^{\star}$, we have

$$
\frac{1}{\rho_{\mathrm{F}} c^{2}} \int_{\Omega_{\mathrm{F}}} p \delta p \mathrm{~d} x+\frac{\lambda^{2}}{c^{2}} \int_{\Omega_{\mathrm{F}}} \varphi \delta p \mathrm{~d} x=0 \quad \forall \delta \varphi \in C_{p}^{\star}
$$


- Multiplying Equation (14f) by a test function $\delta \eta \in C_{\eta}$, we have

$$
\lambda^{2} \rho_{\mathrm{F}} \int_{\Gamma_{\mathrm{A}}} \varphi \delta \eta \mathrm{d} \sigma+\lambda d^{\mathrm{I}} \int_{\Gamma_{\mathrm{A}}} \eta \delta \eta \mathrm{d} \sigma+k^{\mathrm{I}} \int_{\Gamma_{\mathrm{A}}} \eta \delta \eta \mathrm{d} \sigma=0 \quad \forall \delta \varphi \in C_{\eta}
$$

Then, the variational formulation consists in finding $\lambda$ and $(\eta, p, \varphi) \in\left(C_{\eta}, C_{p}^{\star}, C_{\varphi}^{\star}\right)$ satisfying Equations (15)-(17).

The discretization of the preceding formulation leads to the following symmetric matrix equation:

$$
\begin{gathered}
\left(\begin{array}{ccc}
k^{\mathrm{I}} \mathbf{G}_{\eta} & \mathbf{0} & \mathbf{0} \\
\mathbf{0} & \mathbf{K}_{p} & \mathbf{0} \\
\mathbf{0} & \mathbf{0} & \mathbf{0}
\end{array}\right)\left(\begin{array}{c}
\mathbf{H} \\
\mathbf{P} \\
\boldsymbol{\Phi}
\end{array}\right)+\lambda\left(\begin{array}{ccc}
d^{\mathrm{I}} \mathbf{G}_{\eta} & \mathbf{0} & \mathbf{0} \\
\mathbf{0} & \mathbf{0} & \mathbf{0} \\
\mathbf{0} & \mathbf{0} & \mathbf{0}
\end{array}\right)\left(\begin{array}{l}
\mathbf{H} \\
\mathbf{P} \\
\mathbf{\Phi}
\end{array}\right) \\
+\lambda^{2}\left(\begin{array}{ccc}
\mathbf{0} & \mathbf{0} & \mathbf{A} \\
\mathbf{0} & \mathbf{0} & \mathbf{B} \\
\mathbf{A}^{\mathrm{T}} & \mathbf{B}^{\mathrm{T}} & -\mathbf{F}_{\varphi}
\end{array}\right)\left(\begin{array}{l}
\mathbf{H} \\
\mathbf{P} \\
\mathbf{\Phi}
\end{array}\right)=\left(\begin{array}{l}
\mathbf{0} \\
\mathbf{0} \\
\mathbf{0}
\end{array}\right)
\end{gathered}
$$

where $\mathbf{H}, \mathbf{P}$ and $\boldsymbol{\Phi}$ are the vectors of nodal values of $\eta, p$ and $\varphi$, respectively, and the submatrices of Equation (18) are defined by

$$
\begin{aligned}
\rho_{\mathrm{F}} \int_{\Gamma_{\mathrm{A}}} \varphi \delta \eta \mathrm{d} \sigma & \Rightarrow \delta \mathbf{H}^{\mathrm{T}} \mathbf{A} \mathbf{\Phi} \\
\rho_{\mathrm{F}} \int_{\Omega_{\mathrm{F}}} \nabla \varphi \cdot \nabla \delta \varphi \mathrm{d} x & \Rightarrow \delta \mathbf{\Phi}^{\mathrm{T}} \mathbf{F}_{\varphi} \mathbf{\Phi} \\
\frac{1}{\rho_{\mathrm{F}} c^{2}} \int_{\Omega_{\mathrm{F}}} \frac{\partial^{2} p}{\partial t^{2}} \delta p \mathrm{~d} x & \Rightarrow \delta \mathbf{P}^{\mathrm{T}} \mathbf{K}_{p} \ddot{\mathbf{P}} \\
\frac{1}{c^{2}} \int_{\Omega_{\mathrm{F}}} \varphi \delta p \mathrm{~d} \sigma & \Rightarrow \delta \mathbf{P}^{\mathrm{T}} \mathbf{B} \mathbf{\Phi} \\
\int_{\Gamma_{\mathrm{A}}} \eta \delta \eta \mathrm{d} \sigma & \Rightarrow \delta \mathbf{H}^{\mathrm{T}} \mathbf{G}_{\eta} \mathbf{H}
\end{aligned}
$$

We can note that the fluid normal displacement at the absorbing boundary and the fluid pressure can be expressed in terms of fluid potential displacement from the first and the second line of Equation (18):

$$
\begin{aligned}
& \mathbf{H}=\frac{-\lambda^{2}}{k^{\mathrm{I}}+\lambda d^{\mathrm{I}}} \mathbf{G}_{\eta}^{-1} \mathbf{A} \boldsymbol{\Phi} \\
& \mathbf{P}=-\lambda^{2} \mathbf{K}_{p}^{-1} \mathbf{B} \boldsymbol{\Phi}
\end{aligned}
$$


Replacing $\mathbf{H}$ and $\mathbf{P}$ by their expressions, Equation (20), in the third line of Equation (18), we obtain the following nonlinear eigenvalue problem in terms of $\boldsymbol{\Phi}$ :

$$
\left(\mathbf{F}_{\varphi}+\frac{\lambda^{2}}{k^{\mathrm{I}}+\lambda d^{\mathrm{I}}} \mathbf{A}^{\mathrm{T}} \mathbf{G}_{\eta}^{-1} \mathbf{A}+\lambda^{2} \mathbf{B}^{\mathrm{T}} \mathbf{K}_{p}^{-1} \mathbf{B}\right) \boldsymbol{\Phi}=\mathbf{0}
$$

This equation can be obtained directly by using a fluid potential displacement formulation. This formulation has been established by Kehr-Candille and Ohayon [2]. It is clear that this formulation presents the inconvenience that leads to a nonlinear eigenvalue problem which cannot be solved directly.

\section{SYMMETRIC REDUCED MODEL FOR THE DISSIPATIVE ACOUSTIC EIGENVALUE PROBLEM}

In this section, in order to obtain a symmetric reduced model for the dissipative acoustic problem, we propose to eliminate the potential fluid displacement in the symmetric formulation given in the last section (Equation (18)).

First, the third line of Equation (18) is written as

$$
\mathbf{A}^{\mathrm{T}} \mathbf{H}+\mathbf{B}^{\mathrm{T}} \mathbf{P}-\mathbf{F}_{\varphi} \boldsymbol{\Phi}=\mathbf{0}
$$

As the matrix $\mathbf{F}_{\varphi}$ is not singular (due to $\varphi=0$ on $\Gamma_{P}$ ), we can express $\boldsymbol{\Phi}$ in terms of $\mathbf{H}$ and $\mathbf{P}$ from Equation (22):

$$
\boldsymbol{\Phi}=\mathbf{F}_{\varphi}^{-1} \mathbf{A}^{\mathrm{T}} \mathbf{H}+\mathbf{F}_{\varphi}^{-1} \mathbf{B}^{\mathrm{T}} \mathbf{P}
$$

Substituting $\boldsymbol{\Phi}$ by its expression (Equation (23)) in first and second lines of Equation (18), we obtain the following symmetric reduced system in terms of $\eta$ and $p$ :

$$
\begin{gathered}
\left(\begin{array}{cc}
k^{\mathrm{I}} \mathbf{G}_{\eta} & \mathbf{0} \\
\mathbf{0} & \mathbf{K}_{p}
\end{array}\right)\left(\begin{array}{l}
\mathbf{H} \\
\mathbf{P}
\end{array}\right)+\lambda\left(\begin{array}{cc}
d^{\mathrm{I}} \mathbf{G}_{\eta} & \mathbf{0} \\
\mathbf{0} & \mathbf{0}
\end{array}\right)\left(\begin{array}{l}
\mathbf{H} \\
\mathbf{P}
\end{array}\right) \\
+\lambda^{2}\left(\begin{array}{ll}
\mathbf{A} \mathbf{F}_{\varphi}^{-1} \mathbf{A}^{\mathrm{T}} & \mathbf{A F}_{\varphi}^{-1} \mathbf{B}^{\mathrm{T}} \\
\mathbf{B F}_{\varphi}^{-1} \mathbf{A}^{\mathrm{T}} & \mathbf{B} \mathbf{F}_{\varphi}^{-1} \mathbf{B}^{\mathrm{T}}
\end{array}\right)\left(\begin{array}{l}
\mathbf{H} \\
\mathbf{P}
\end{array}\right)=\left(\begin{array}{l}
\mathbf{0} \\
\mathbf{0}
\end{array}\right)
\end{gathered}
$$

\subsection{Case in which $\Gamma_{P}=\emptyset$}

In this case, Equation (14d) is removed from the local equations (14a)-(14f). The associated variational formulation is the same as Equations (15)-(17) with admissible spaces without any constraints. In Equation (18), the matrix $\mathbf{F}_{\varphi}$ is now singular and consequently $\boldsymbol{\Phi}$ cannot be eliminated. In order to reduce the problem, we use the procedure described by Morand and Ohayon for elasto-acoustic problem [6] as detailed below.

Let us define a partitioning of $\boldsymbol{\Phi}$ by letting $\boldsymbol{\Phi}^{\mathrm{T}}=\left[\begin{array}{ll}\Phi_{1} & \boldsymbol{\Phi}_{2}^{\mathrm{T}}\end{array}\right]$ where $\Phi_{1}$ denotes a particular component of $\boldsymbol{\Phi}$ (here, the first), which induces the following partitioning of the matrices $\mathbf{A}$, 
$\mathbf{B}$ and $\mathbf{F}_{\varphi}$ in Equation (18):

$$
\begin{gathered}
\left(\begin{array}{cccc}
k^{\mathrm{I}} \mathbf{G}_{\eta} & \mathbf{0} & \mathbf{0} & \mathbf{0} \\
\mathbf{0} & \mathbf{K}_{p} & \mathbf{0} & \mathbf{0} \\
\mathbf{0} & \mathbf{0} & 0 & \mathbf{0} \\
\mathbf{0} & \mathbf{0} & \mathbf{0} & \mathbf{0}
\end{array}\right)\left(\begin{array}{c}
\mathbf{H} \\
\mathbf{P} \\
\Phi_{1} \\
\boldsymbol{\Phi}_{2}
\end{array}\right)+\lambda\left(\begin{array}{cccc}
d^{\mathrm{I}} \mathbf{G}_{\eta} & \mathbf{0} & \mathbf{0} & \mathbf{0} \\
\mathbf{0} & \mathbf{0} & \mathbf{0} & \mathbf{0} \\
\mathbf{0} & \mathbf{0} & 0 & \mathbf{0} \\
\mathbf{0} & \mathbf{0} & \mathbf{0} & \mathbf{0}
\end{array}\right)\left(\begin{array}{c}
\mathbf{H} \\
\mathbf{P} \\
\Phi_{1} \\
\boldsymbol{\Phi}_{2}
\end{array}\right) \\
+\lambda^{2}\left(\begin{array}{ccccc}
\mathbf{0} & \mathbf{0} & \mathbf{A}_{1} & \mathbf{A}_{2} \\
\mathbf{0} & \mathbf{0} & \mathbf{B}_{1} & \mathbf{B}_{2} \\
\mathbf{A}_{1}^{\mathrm{T}} & \mathbf{B}_{1}^{\mathrm{T}} & -F_{\varphi 11} & -\mathbf{F}_{\varphi 12} \\
\mathbf{A}_{2}^{\mathrm{T}} & \mathbf{B}_{2}^{\mathrm{T}} & -\mathbf{F}_{\varphi 21} & -\mathbf{F}_{\varphi 22}
\end{array}\right)\left(\begin{array}{c}
\mathbf{H} \\
\mathbf{P} \\
\Phi_{1} \\
\boldsymbol{\Phi}_{2}
\end{array}\right)=\left(\begin{array}{l}
\mathbf{0} \\
\mathbf{0} \\
0 \\
\mathbf{0}
\end{array}\right)
\end{gathered}
$$

$\mathbf{F}_{\varphi 22}$ being nonsingular, $\boldsymbol{\Phi}_{2}$ can be expressed in terms of $\mathbf{P}, \mathbf{H}$ and $\Phi_{1}$ using the fourth line of Equation (25):

$$
\boldsymbol{\Phi}_{2}=-\mathbf{F}_{\varphi 22}^{-1} \mathbf{F}_{\varphi 21} \Phi_{1}+\mathbf{F}_{\varphi 22}^{-1} \mathbf{A}_{2}^{\mathrm{T}} \mathbf{H}+\mathbf{F}_{\varphi 22}^{-1} \mathbf{B}_{2}^{\mathrm{T}} \mathbf{P}
$$

Substituting this relation for the remaining, the third line of Equation (25) becomes:

$$
\left(\mathbf{A}_{1}^{\mathrm{T}}-\mathbf{F}_{\varphi 12} \mathbf{F}_{\varphi 22}^{-1} \mathbf{A}_{2}^{\mathrm{T}}\right) \mathbf{H}+\left(\mathbf{B}_{1}^{\mathrm{T}}-\mathbf{F}_{\varphi 12} \mathbf{F}_{\varphi 22}^{-1} \mathbf{B}_{2}^{\mathrm{T}}\right) \mathbf{P}=\left(F_{\varphi 11}-\mathbf{F}_{\varphi 12} \mathbf{F}_{\varphi 22}^{-1} \mathbf{F}_{\varphi 21}\right) \Phi_{1}
$$

It can be shown that [6]:

$$
F_{\varphi 11}-\mathbf{F}_{\varphi 12} \mathbf{F}_{\varphi 22}^{-1} \mathbf{F}_{\varphi 12}^{\mathrm{T}}=0
$$

Thus, Equation (27) can be written as

$$
\mathbf{a}^{\mathrm{T}} \mathbf{H}+\mathbf{b}^{\mathrm{T}} \mathbf{P}=0
$$

where matrices $\mathbf{a}$ and $\mathbf{b}$ are defined by

$$
\begin{array}{r}
\mathbf{a}^{\mathrm{T}}=\mathbf{A}_{1}^{\mathrm{T}}-\mathbf{F}_{\varphi 12} \mathbf{F}_{\varphi 22}^{-1} \mathbf{A}_{2}^{\mathrm{T}} \\
\mathbf{b}^{\mathrm{T}}=\mathbf{B}_{1}^{\mathrm{T}}-\mathbf{F}_{\varphi 12} \mathbf{F}_{\varphi 22}^{-1} \mathbf{B}_{2}^{\mathrm{T}}
\end{array}
$$

Finally, replacing $\boldsymbol{\Phi}_{2}$ by its expression (26) in the two first lines of Equation (25) and using Equation (29), the following matrix system is obtained:

$$
\begin{gathered}
\left(\begin{array}{ccc}
k^{\mathrm{I}} \mathbf{G}_{\eta} & \mathbf{0} & \mathbf{0} \\
\mathbf{0} & \mathbf{K}_{p} & \mathbf{0} \\
\mathbf{0} & \mathbf{0} & 0
\end{array}\right)\left(\begin{array}{c}
\mathbf{H} \\
\mathbf{P} \\
\Phi_{1}
\end{array}\right)+\lambda\left(\begin{array}{ccc}
d^{\mathrm{I}} \mathbf{G}_{\eta} & \mathbf{0} & \mathbf{0} \\
\mathbf{0} & \mathbf{0} & \mathbf{0} \\
\mathbf{0} & \mathbf{0} & 0
\end{array}\right)\left(\begin{array}{c}
\mathbf{H} \\
\mathbf{P} \\
\Phi_{1}
\end{array}\right) \\
+\lambda^{2}\left(\begin{array}{ccc}
\mathbf{A}_{2} \mathbf{F}_{\varphi 22}^{-1} \mathbf{A}_{2}^{\mathrm{T}} & \mathbf{A}_{2} \mathbf{F}_{\varphi 22}^{-1} \mathbf{B}_{2}^{\mathrm{T}} & \mathbf{a} \\
\mathbf{B}_{2} \mathbf{F}_{\varphi 22}^{-1} \mathbf{A}_{2}^{\mathrm{T}} & \mathbf{B}_{2} \mathbf{F}_{\varphi 22}^{-1} \mathbf{B}_{2}^{\mathrm{T}} & \mathbf{b} \\
\mathbf{a}^{\mathrm{T}} & \mathbf{b}^{\mathrm{T}} & 0
\end{array}\right)\left(\begin{array}{c}
\mathbf{H} \\
\mathbf{P} \\
\Phi_{1}
\end{array}\right)=\left(\begin{array}{c}
\mathbf{0} \\
\mathbf{0} \\
0
\end{array}\right)
\end{gathered}
$$


It is important to note that previous reduction is not an approximation but a rigorous condensation procedure.

\subsection{Reduced eigenvalue problem}

The reduced eigenvalue problem (Equation (24) or (31) for the case $\Gamma_{P}=\emptyset$ ) corresponds in fact to an indirect (via the intermediate displacement potential unknown field) symmetric formulation in terms of $\eta$ and $p$. In a condensed form, these matrix equations can be written as

$$
\left(\mathscr{K}+\lambda \mathscr{D}+\lambda^{2} \mathscr{M}\right) \mathbf{X}=\mathbf{0}
$$

In practice, this second-order eigenvalue problem can be solved by transforming it into a system of $2 n$ first-order equations by complementing it with the equality $\lambda \mathscr{M} \mathbf{X}-\lambda \mathscr{M} \mathbf{X}=\mathbf{0}$, where $n$ is the number of degrees of freedom (d.o.f.) in the system. The extended spectral equation takes the canonical form:

$$
\left[\left(\begin{array}{cc}
\mathscr{K} & \mathbf{0} \\
\mathbf{0} & -\mathscr{M}
\end{array}\right)+\lambda\left(\begin{array}{cc}
\mathscr{D} & \mathscr{M} \\
\mathscr{M} & \mathbf{0}
\end{array}\right)\right]\left(\begin{array}{l}
\mathbf{X} \\
\lambda \mathbf{X}
\end{array}\right)=\mathbf{0}
$$

The matrices $\mathscr{M}, \mathscr{D}$ and $\mathscr{K}$ being real symmetric, complex eigenvalues come in conjugate pairs associated with conjugate pairs of mode shapes.

\section{NUMERICAL EXAMPLES}

In this section, numerical results are obtained via a specific Matlab code especially developed for this project.

\subsection{Modal analysis of a square cavity with a damping wall}

In order to validate the finite element implementation of the proposed damping formulation, we firstly consider the spectral problem of a square cavity of size $(1 \mathrm{~m} \times 1 \mathrm{~m})$ completely filled with air and with an absorbing wall (Figure 2). In this example, initially proposed in Reference [3], the following data are used: $\rho_{\mathrm{F}}=1 \mathrm{~kg} / \mathrm{m}^{3}, c=340 \mathrm{~m} / \mathrm{s}, k^{\mathrm{I}}=5 \times 10^{6} \mathrm{~Pa} / \mathrm{m}$ and $d^{\mathrm{I}}=50 \mathrm{~Pa} \mathrm{~s} / \mathrm{m}$. The last two parameters are average impedance coefficients corresponding to a typical acoustic insulating fabric (a Johns Manville glass wool) in the frequency range $(50-500 \mathrm{~Hz})$ (see Figure 3).

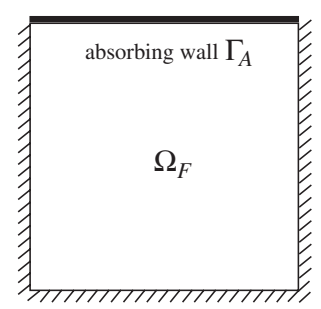

Figure 2. Fluid in square rigid cavity with an absorbing wall. 


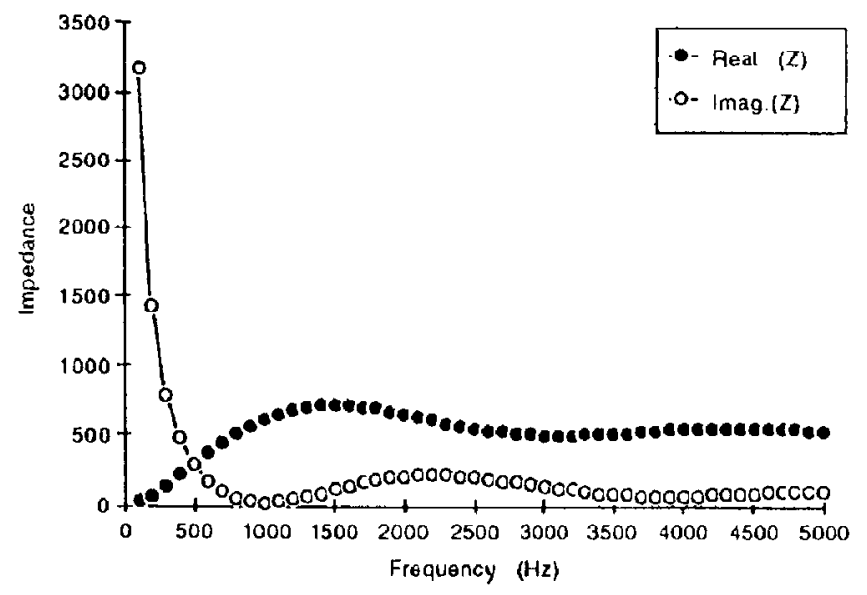

Figure 3. Acoustic impedance for Johns Manville glass wool [2].

Table I. Frequencies $(\mathrm{Hz})$ of a square rigid cavity with an absorbing wall.

\begin{tabular}{lccccc}
\hline Undamped & & & \multicolumn{3}{c}{ Damped } \\
\cline { 1 - 2 } \cline { 4 - 6 } 961 d.o.f. & Exact $[10]$ & & 121 d.o.f. & 961 d.o.f. & Exact [3] \\
\hline 170.078 & $f_{01}=170$ & & $166.790-0.040 \mathrm{i}$ & $166.233-0.039 \mathrm{i}$ & $166.165-0.039 \mathrm{i}$ \\
170.078 & $f_{10}=170$ & & $168.737-0.023 \mathrm{i}$ & $167.982-0.023 \mathrm{i}$ & $167.919-0.023 \mathrm{i}$ \\
240.526 & $f_{11}=240.416$ & & $236.019-0.078 \mathrm{i}$ & $235.073-0.079 \mathrm{i}$ & $234.957-0.079 \mathrm{i}$ \\
340.622 & $f_{02}=340$ & & $337.365-0.168 \mathrm{i}$ & $332.910-0.155 \mathrm{i}$ & $332.370-0.154 \mathrm{i}$ \\
340.622 & $f_{20}=340$ & & $341.094-0.123 \mathrm{i}$ & $335.327-0.141 \mathrm{i}$ & $334.720-0.144 \mathrm{i}$ \\
\hline
\end{tabular}

In Table I, the first five eigenfrequencies (in $\mathrm{Hz}$ ) with uniform meshes (quadrangular element) and with increasing number of d.o.f. are presented.

The first and second columns present the eigenfrequencies of the rigid cavity computed from the pressure formulation and compared to the exact solution given in Reference [10] for a rectangular cavity of lengths $A$ and $B$ (here, $A=B=1 \mathrm{~m}$ ):

$$
f_{n m}(\mathrm{~Hz})=\frac{c}{2} \sqrt{\left(\frac{n}{A}\right)^{2}+\left(\frac{m}{B}\right)^{2}}, \quad n, m=0,1,2, \ldots,(n+m \neq 0)
$$

This comparison enables us to validate the development of the fluid finite element. The three other columns correspond to the complex frequencies of the damping cavity computed from the $(\eta, p)$-symmetric reduced formulation (Equation (31)) and compared to exact solution (last column) given by Bermúdez and Rodríguez [3]. A good agreement between exact and computed values can be observed even for the coarse mesh. In this example, the imaginary part of the frequencies derives from dashpot dissipation. Moreover, the difference between the real part (damped case) and the real value (undamped case) of the frequencies is due to the spring effect. 
It can be noted that the first five mode shapes are different in the damped and undamped cases, except for the third one (Figures 4-6). This is due to the lack of symmetry appearing in the problem with one dissipative wall.

\subsection{Modal analysis of a damping rectangular cavity}

The second example concerns a $2 \mathrm{D}$ rectangular rigid cavity $(A=1 \mathrm{~m}, B=0.75 \mathrm{~m})$ filled with air $\left(\rho_{f}=1 \mathrm{~kg} / \mathrm{m}^{3}, c=340 \mathrm{~m} / \mathrm{s}\right)$. As in the previous example, a thin layer of absorbing material covers the top wall of the cavity. Here, the parameters of the Kelvin-Voigt model are: $k^{\mathrm{I}}=5 \times 10^{4} \mathrm{~Pa} / \mathrm{m}$ and $d^{\mathrm{I}}=200 \mathrm{~Pa} \mathrm{~s} / \mathrm{m}$.

Figures 7-9 show the mode shapes and Table II gives the eigenvalues of the discretized problem given in Equation (31). We have computed them with different successively refined uniform meshes. In the last column of the table, the exact eigenvalues given by Bermúdez et al. [9] are included. As it can be seen, our solution converges to the exact one when refining the mesh. In this example, the imaginary part of frequencies is much more important than in the previous example. Here, the real and imaginary parts of the frequencies can have the same order of magnitude for some of the first modes.
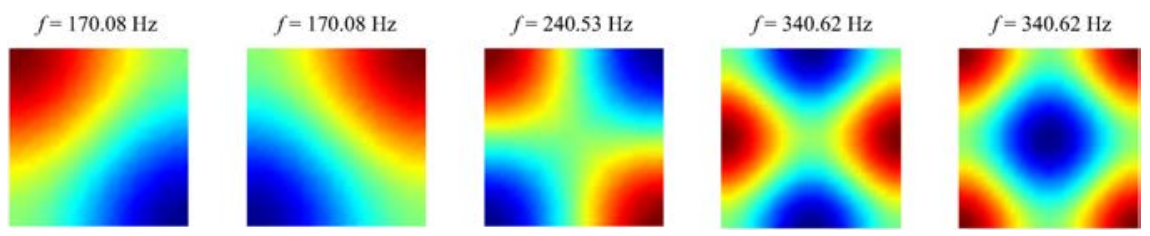

Figure 4. First five acoustic modes for the square rigid cavity.
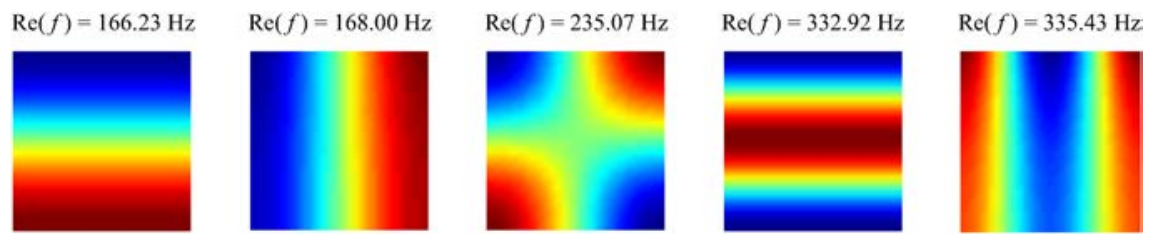

Figure 5. First five acoustic modes for the square cavity with an absorbing wall (real part).
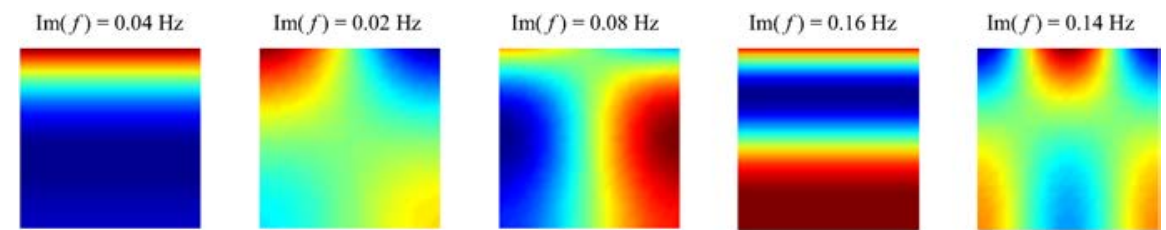

Figure 6. First five acoustic modes for the square cavity with an absorbing wall (imaginary part). 

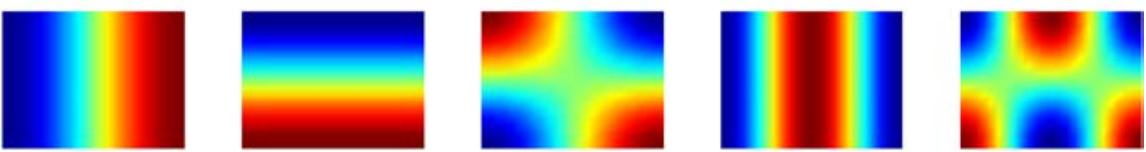

Figure 7. First five acoustic modes for a rectangular rigid cavity.

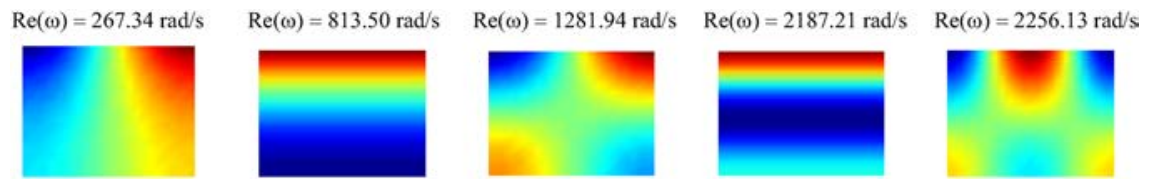

Figure 8. First five acoustic modes for the rectangular cavity with an absorbing wall (real part).

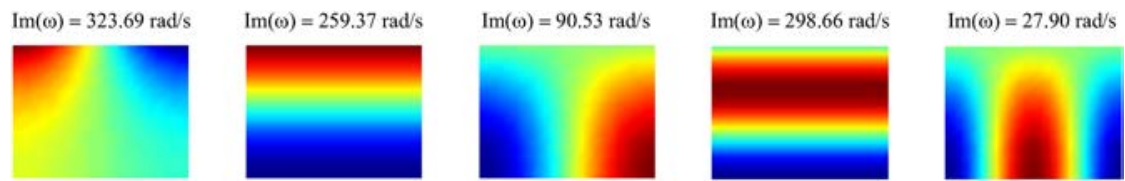

Figure 9. First five acoustic modes for the rectangular cavity with an absorbing wall (imaginary part).

Table II. Frequencies ( $\mathrm{rad} / \mathrm{s})$ of a rectangular rigid cavity with an absorbing wall.

\begin{tabular}{lccc}
\hline 475 d.o.f. & 825 d.o.f. & 1271 d.o.f. & Exact [9] \\
\hline $267.34+323.69 \mathrm{i}$ & $267.48+322.38 \mathrm{i}$ & $267.54+321.78 \mathrm{i}$ & $267.65+320.71 \mathrm{i}$ \\
$813.50+259.37 \mathrm{i}$ & $813.41+259.30 \mathrm{i}$ & $813.36+259.27 \mathrm{i}$ & $813.29+259.21 \mathrm{i}$ \\
$1281.94+90.53 \mathrm{i}$ & $1281.68+90.28 \mathrm{i}$ & $1281.56+90.16 \mathrm{i}$ & $1281.35+89.95 \mathrm{i}$ \\
$2187.21+298.66 \mathrm{i}$ & $2184.56+298.03 \mathrm{i}$ & $2183.33+297.74 \mathrm{i}$ & $2181.15+297.21 \mathrm{i}$ \\
$2256.13+27.90 \mathrm{i}$ & $2253.63+027.66 \mathrm{i}$ & $2252.47+27.56 \mathrm{i}$ & $2250.41+27.37 \mathrm{i}$ \\
$2415.15+239.82 \mathrm{i}$ & $2412.54+238.45 \mathrm{i}$ & $2411.34+237.82 \mathrm{i}$ & $2409.21+236.70 \mathrm{i}$ \\
$3032.04+147.80 \mathrm{i}$ & $3028.395+145.74 \mathrm{i}$ & $3026.69+144.80 \mathrm{i}$ & $3023.68+143.16 \mathrm{i}$ \\
$3302.15+13.24 \mathrm{i}$ & $3293.36+12.99 \mathrm{i}$ & $3289.29+12.88 \mathrm{i}$ & $3282.07+12.69 \mathrm{i}$ \\
$3616.68+306.56 \mathrm{i}$ & $3604.30+304.85 \mathrm{i}$ & $3598.59+304.05 \mathrm{i}$ & $3588.43+302.60 \mathrm{i}$ \\
$3765.36+281.50 \mathrm{i}$ & $3753.29+278.85 \mathrm{i}$ & $3747.71+277.61 \mathrm{i}$ & $3737.81+275.41 \mathrm{i}$
\end{tabular}

\subsection{Dissipative acoustic pipe submitted to transient pressure loads}

In this last example, a pressure load is applied to the left edge of a straight pipe $(A=5 \mathrm{~m}$, $B=0.5 \mathrm{~m}$ ) containing air and with an absorption wall at the right edge (Figure 10). The damping parameters are chosen in order to attenuate the vibration amplitudes $\left(k^{\mathrm{I}}=1 \times 10^{6} \mathrm{~Pa} / \mathrm{m}\right.$ and $d^{\mathrm{I}}=500 \mathrm{~Pa} \mathrm{~s} / \mathrm{m}$ ) without shifting the frequencies in a too significant way. 


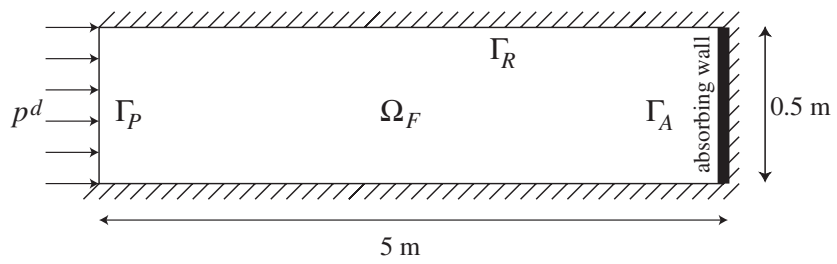

Figure 10. Straight pipe with a pressure pulse applied at the left end.

Table III. Frequencies $(\mathrm{Hz})$ of the pipe.

\begin{tabular}{|c|c|c|}
\hline \multicolumn{2}{|c|}{ Rigid cavity } & \multirow{2}{*}{ 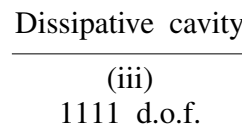 } \\
\hline $\begin{array}{l}\text { (i) } \\
1111 \text { d of. }\end{array}$ & $\begin{array}{l}\text { (ii) } \\
1100 \text { d.o.f. }\end{array}$ & \\
\hline & & \\
\hline 34.00 & 17.00 & $16.62-0.02 \mathrm{i}$ \\
\hline 68.01 & 51.00 & $49.88-0.17 \mathrm{i}$ \\
\hline 102.04 & 85.02 & $83.23-0.45 \mathrm{i}$ \\
\hline 136.09 & 119.06 & $116.69-0.82 \mathrm{i}$ \\
\hline 170.17 & 153.13 & $150.28-1.25 \mathrm{i}$ \\
\hline 204.30 & 187.23 & $184.01-1.70 \mathrm{i}$ \\
\hline 238.48 & 221.38 & $217.87-2.15 \mathrm{i}$ \\
\hline 272.72 & 255.59 & $251.86-2.59 \mathrm{i}$ \\
\hline 307.02 & 289.86 & $285.96-3.02 \mathrm{i}$ \\
\hline 341.40 & 324.20 & $320.19-3.42 \mathrm{i}$ \\
\hline
\end{tabular}

Table III shows the eigenvalues of the fluid for various boundary conditions: (i) rigid boundary conditions along all edges, (ii) null pressure on $\Gamma_{P}$ and rigid boundary elsewhere, (iii) null pressure on $\Gamma_{P}$, absorbing wall on $\Gamma_{\mathrm{A}}$, and rigid boundary on $\Gamma_{\mathrm{R}}$. As it can be seen, the difference between frequencies in the first (1111 d.o.f.) and second (1100 d.o.f.) columns is due to the elimination of the fixed d.o.f. on the left edge where a pressure load is applied. In the third column, where additional normal fluid displacements are taken into account, the real parts of the complex frequencies of the dissipative pipe are close to the frequencies of the rigid pipe. Moreover, the imaginary parts, which are small compared to the real parts, are associated with the damping effect.

Equation (18) can be transposed in time domain in a straithforward manner, leading to a standard time second-order matrix system. Let us partition the vector of nodal pressures into $\overline{\mathbf{P}}$ (where the pressure is prescribed) and $\mathbf{P}$ (where the pressure is unknown). Similarly, we divide the fluid potential displacement into $\overline{\boldsymbol{\Phi}}$ and $\boldsymbol{\Phi}$, where $\overline{\boldsymbol{\Phi}}$ is given from $\overline{\mathbf{P}}$ using the following relation:

$$
\frac{\partial^{2} \varphi}{\partial t^{2}}=-\frac{p}{\rho_{\mathrm{F}}} \quad \text { in } \Omega_{\mathrm{F}}
$$

deduced from the linearized Euler equations. 
With this separation into known and unknown components, Equation (18) can be transposed in time domain as follows:

$$
\begin{gathered}
\left(\begin{array}{ccccc}
k^{\mathrm{I}} \mathbf{G}_{\eta} & \mathbf{0} & \mathbf{0} & \mathbf{0} & \mathbf{0} \\
\mathbf{0} & \mathbf{K}_{p} & \mathbf{K}_{p \bar{p}} & \mathbf{0} & \mathbf{0} \\
\mathbf{0} & \mathbf{K}_{\bar{p} p} & \mathbf{K}_{\bar{p}} & \mathbf{0} & \mathbf{0} \\
\mathbf{0} & \mathbf{0} & \mathbf{0} & \mathbf{0} & \mathbf{0} \\
\mathbf{0} & \mathbf{0} & \mathbf{0} & \mathbf{0} & \mathbf{0}
\end{array}\right)\left(\begin{array}{c}
\mathbf{H} \\
\mathbf{P} \\
\overline{\mathbf{P}} \\
\boldsymbol{\Phi} \\
\overline{\mathbf{\Phi}}
\end{array}\right)+\left(\begin{array}{ccccc}
d^{\mathrm{I}} \mathbf{G}_{\eta} & \mathbf{0} & \mathbf{0} & \mathbf{0} & \mathbf{0} \\
\mathbf{0} & \mathbf{0} & \mathbf{0} & \mathbf{0} & \mathbf{0} \\
\mathbf{0} & \mathbf{0} & \mathbf{0} & \mathbf{0} & \mathbf{0} \\
\mathbf{0} & \mathbf{0} & \mathbf{0} & \mathbf{0} & \mathbf{0} \\
\mathbf{0} & \mathbf{0} & \mathbf{0} & \mathbf{0} & \mathbf{0}
\end{array}\right)\left(\begin{array}{c}
\dot{\mathbf{H}} \\
\dot{\mathbf{P}} \\
\dot{\overline{\mathbf{P}}} \\
\dot{\boldsymbol{\Phi}} \\
\dot{\overline{\mathbf{\Phi}}}
\end{array}\right) \\
+\left(\begin{array}{ccccc}
\mathbf{0} & \mathbf{0} & \mathbf{0} & \mathbf{A}_{\eta \varphi} & \mathbf{A}_{\eta \bar{\varphi}} \\
\mathbf{0} & \mathbf{0} & \mathbf{0} & \mathbf{B}_{p \varphi} & \mathbf{B}_{p \bar{\varphi}} \\
\mathbf{0} & \mathbf{0} & \mathbf{0} & \mathbf{B}_{\bar{p} \varphi} & \mathbf{B}_{\bar{p} \bar{\varphi}} \\
\mathbf{A}_{\eta \varphi}^{\mathrm{T}} & \mathbf{B}_{p \varphi}^{\mathrm{T}} & \mathbf{B}_{\bar{p} \varphi}^{\mathrm{T}} & -\mathbf{F}_{\varphi} & -\mathbf{F}_{\varphi \bar{\varphi}} \\
\mathbf{A}_{\eta \bar{\varphi}}^{\mathrm{T}} & \mathbf{B}_{p \bar{\varphi}}^{\mathrm{T}} & \mathbf{B}_{\bar{p}}^{\mathrm{T}} \bar{\varphi} & -\mathbf{F}_{\bar{\varphi} \varphi} & -\mathbf{F}_{\bar{\varphi}}
\end{array}\right)\left(\begin{array}{c}
\ddot{\mathbf{H}} \\
\ddot{\mathbf{P}} \\
\ddot{\mathbf{P}} \\
\ddot{\mathbf{\Phi}} \\
\ddot{\mathbf{\Phi}}
\end{array}\right)=\left(\begin{array}{c}
\mathbf{0} \\
\mathbf{0} \\
\mathbf{0} \\
\mathbf{0} \\
\mathbf{0}
\end{array}\right)
\end{gathered}
$$

The third and the fifth lines of the previous matrix system are eliminated because they correspond to the known nodal values $\overline{\mathbf{P}}$ and $\overline{\boldsymbol{\Phi}}$. Using the fourth line of Equation (36), $\ddot{\boldsymbol{\Phi}}$ can be expressed in terms of other variables in the following form:

$$
\ddot{\boldsymbol{\Phi}}=\mathbf{F}_{\varphi}^{-1}\left(\mathbf{A}_{\eta \varphi}^{\mathrm{T}} \ddot{\mathbf{H}}+\mathbf{B}_{p \varphi}^{\mathrm{T}} \ddot{\mathbf{P}}+\mathbf{B}_{\bar{p} \varphi}^{\mathrm{T}} \ddot{\overline{\mathbf{P}}}-\mathbf{F}_{\varphi \bar{\varphi}} \ddot{\overline{\mathbf{\Phi}}}\right)
$$

Finally, replacing $\ddot{\boldsymbol{\Phi}}$ by its expression (Equation (37)) in the first and second lines of Equation (36) and writing all of the known variables on the right-hand side, we obtain the following symmetric matrix system:

$$
\begin{aligned}
& \left(\begin{array}{cc}
k^{\mathrm{I}} \mathbf{G}_{\eta} & \mathbf{0} \\
\mathbf{0} & \mathbf{K}_{p}
\end{array}\right)\left(\begin{array}{c}
\mathbf{H} \\
\mathbf{P}
\end{array}\right)+\left(\begin{array}{cc}
d^{\mathrm{I}} \mathbf{G}_{\eta} & \mathbf{0} \\
\mathbf{0} & \mathbf{0}
\end{array}\right)\left(\begin{array}{c}
\dot{\mathbf{H}} \\
\dot{\mathbf{P}}
\end{array}\right) \\
& +\left(\begin{array}{ll}
\mathbf{A}_{\eta \varphi} \mathbf{F}_{\varphi}^{-1} \mathbf{A}_{\eta \varphi}^{\mathrm{T}} & \mathbf{A}_{\eta \varphi} \mathbf{F}_{\varphi}^{-1} \mathbf{B}_{p \varphi}^{\mathrm{T}} \\
\mathbf{B}_{p \varphi} \mathbf{F}_{\varphi}^{-1} \mathbf{A}_{\eta \varphi}^{\mathrm{T}} & \mathbf{B}_{p \varphi} \mathbf{F}_{\varphi}^{-1} \mathbf{B}_{p \varphi}^{\mathrm{T}}
\end{array}\right)\left(\begin{array}{l}
\ddot{\mathbf{H}} \\
\ddot{\mathbf{P}}
\end{array}\right)=\left(\begin{array}{c}
\mathbf{F}_{\eta} \\
\mathbf{F}_{p}
\end{array}\right)
\end{aligned}
$$

where $\mathbf{F}_{\eta}$ and $\mathbf{F}_{p}$ are given by

$$
\begin{aligned}
& \mathbf{F}_{\eta}=\left(-\mathbf{A}_{\eta \bar{\varphi}}+\mathbf{A}_{\eta \varphi} \mathbf{F}_{\varphi}^{-1} \mathbf{F}_{\varphi \bar{\varphi}}\right) \ddot{\overline{\mathbf{\Phi}}}-\mathbf{A}_{\eta \varphi} \mathbf{F}_{\varphi}^{-1} \mathbf{B}_{\bar{p} \varphi}^{\mathrm{T}} \ddot{\overline{\mathbf{P}}} \\
& \mathbf{F}_{p}=\left(-\mathbf{B}_{p \bar{\varphi}}+\mathbf{B}_{p \varphi} \mathbf{F}_{\varphi}^{-1} \mathbf{F}_{\varphi \bar{\varphi}}\right) \ddot{\overline{\mathbf{\Phi}}}-\mathbf{B}_{p \varphi} \mathbf{F}_{\varphi}^{-1} \mathbf{B}_{\bar{p} \varphi}^{\mathrm{T}} \ddot{\overline{\mathbf{P}}}-\mathbf{K}_{p \bar{p}} \overline{\mathbf{P}}
\end{aligned}
$$

and with: $\overline{\overline{\mathbf{\Phi}}}-\mathbf{P} † \rho_{\mathrm{F}}$ (see Equation (35)). 
It should be noted that the matrix system (38) can be directly integrated in time domain. However, it can be costly because the mass matrix is fully populated. Alternatively, it can used in its spectral form in a modal reduction procedure as described below.

7.3.1. Modal reduction. The superposition method is applied here in order to reduce the finite element model proposed in Equation (38), which can be written in condensed form:

$$
\mathscr{M} \ddot{\mathbf{X}}+\mathscr{D} \dot{\mathbf{X}}+\mathscr{K} \mathbf{X}=\mathscr{F}
$$

Therefore, Equation (40) has to be transformed into modal or generalized coordinates of much small degrees of freedom. The undamped modal data can be obtained by solving the real eigenvalue problem:

$$
\mathscr{K}\{\phi\}+\lambda^{2} \mathscr{M}\{\phi\}=\mathbf{0}
$$

The modal transformation and truncation of matrix system (Equation (40)) is accomplished by using ' $r$ ' columns of the modal matrix $[\phi]$ (' $r$ ' represents the number of the first most significant vibration modes of the fluid). As a result, our differential system is reduced to ' $r$ ' d.o.f., which are solved using the Newmark numerical integration technique:

$$
\mathscr{M}^{\mathrm{R}} \ddot{\mathbf{X}}^{\mathrm{R}}+\mathscr{D}^{\mathrm{R}} \dot{\mathbf{X}}^{\mathrm{R}}+\mathscr{K}^{\mathrm{R}} \mathbf{X}^{\mathrm{R}}=\mathscr{F}^{\mathrm{R}}
$$

where, the superscript $\mathrm{R}$ represents the reduced system. We then transferred the modal solutions back to the original coordinate system using the following transformation:

$$
\mathbf{X}=[\phi]^{\mathrm{R}} \mathbf{X}^{\mathrm{R}}
$$

7.3.2. Numerical results. In this last section, transient response of the damped pipe is computed using a direct time integration method applied (i) to the global unsymmetric $(\eta, p)$ system (Equation (9)), and (ii) to the reduced symmetric system (Equation (42)). Two types of excitation are considered:

1. Pressure pulse:

$$
p^{d}(t)= \begin{cases}p_{0} \sin \left(\pi t / T_{1}\right) & \text { for } 0 \leqslant t \leqslant T_{1} \\ 0 & \text { for } t \geqslant T_{1}\end{cases}
$$

\section{Harmonic excitation:}

$$
p^{d}(t)=p_{0} \sin \left(2 \pi t / T_{1}\right)
$$

where $p_{0}=1000 \mathrm{~Pa}$ is the amplitude of the pressure load, $T_{1}=20 \mu \mathrm{s}$ is the pressure pulse length (case 1) or the period of excitation (case 2).

Figure 11 shows time and frequency responses of the dissipative acoustic pipe in a point $\mathrm{M}$ $(2.5 \mathrm{~m}, 0.4 \mathrm{~m})$ when a pressure pulse is applied to the left edge. As shown in Figure 11(a), the pressure levels of the undamped system have been significantly reduced due to the damping effect. Figure 11(b) presents the decrease in the peak levels that were obtained using the absorption wall at the right edge of the pipe. These figures clearly show that there is a substantial reduction in pressure amplitude due to the addition of the damping interface. 

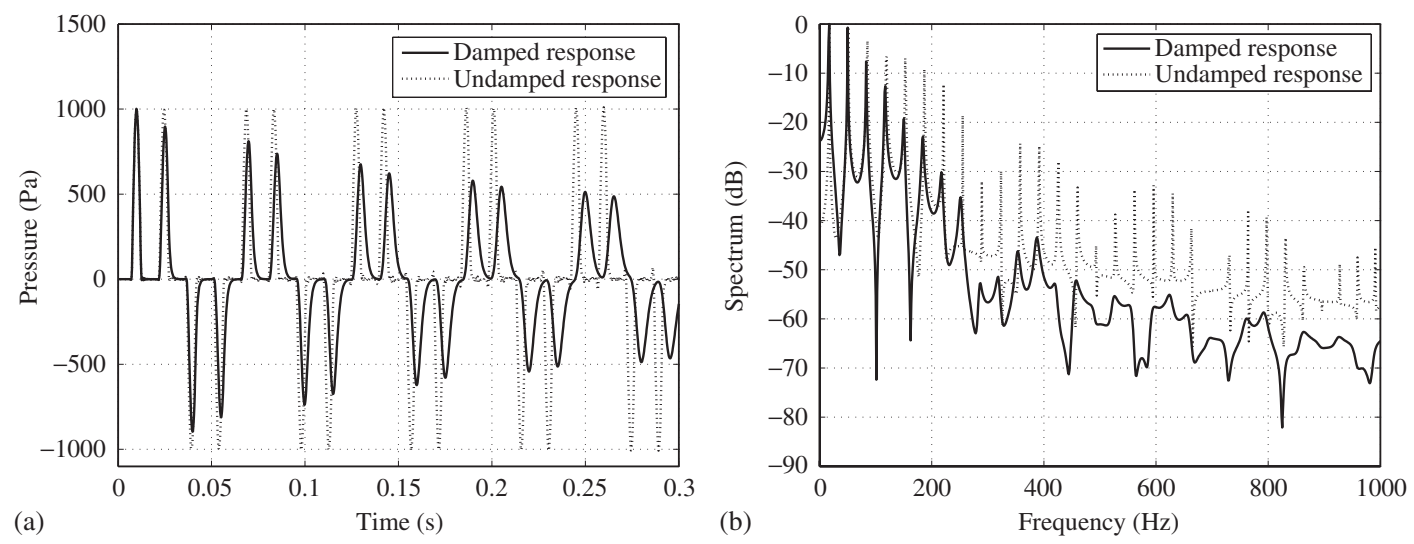

Figure 11. Pipe response to pressure pulse in the damped and undamped case: (a) in time domain; and (b) in frequency domain.
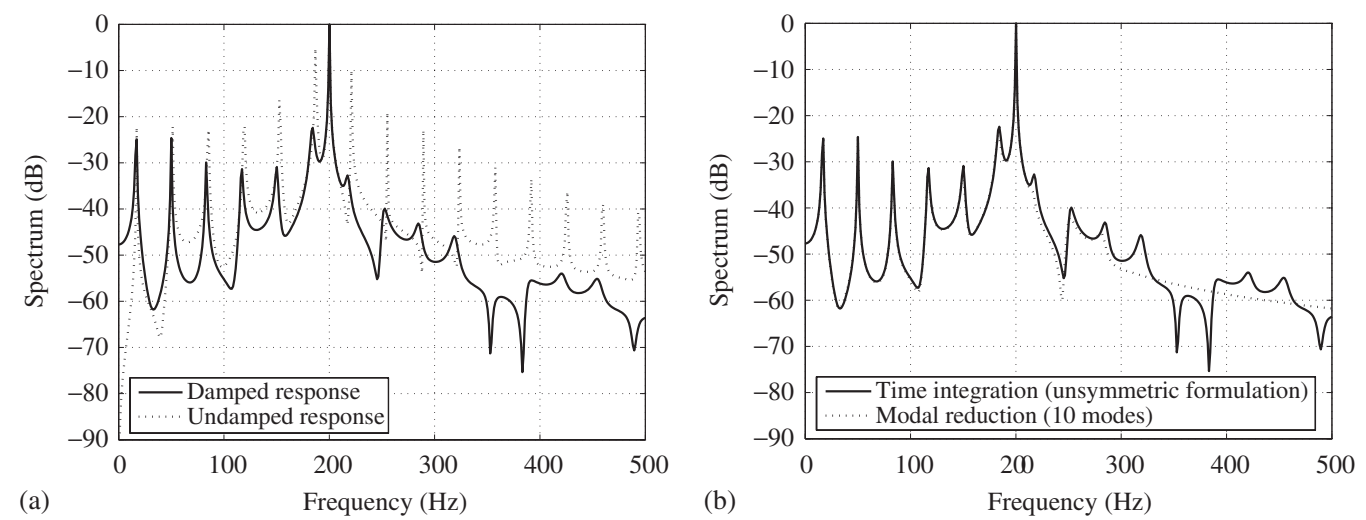

Figure 12. Frequency pipe responses to sinusoidal pressure: (a) damped and undamped case; and (b) time integration (unsymmetric formulation) and modal reduction (symmetric formulation with 10 modes).

Figure 12(a) illustrates the effect of the damping wall in the case of harmonic excitation. The addition of an absorbing boundary caused a small shift in some of the resonant frequencies of pipe except for the seventh, which corresponds to excitation frequency $(200 \mathrm{~Hz})$. This shift is due to the spring effect of the Kelvin-Voigt model. This figure also indicates that the damping significantly reduced the first resonant peaks, with the largest reductions achieved after the excitation peak. Figure 12(b) compares the noise levels for the damped pipe using a time integration method applied to the global unsymmetric $(\eta, p)$ system or a modal reduction technique applied to the symmetric $(\eta, p)$ formulation presented in Section 7.3.1 using only the 10 first fluid modes. The result improves the procedure of symmetrisation and the performance of modal reduction method. 


\section{CONCLUSION}

This paper presents a new formulation for analysis of internal acoustic problem with absorbing walls which is based on the fluid pressure field and the normal fluid displacement on the damped walls. Local equations and corresponding variational formulations leading to a reduced-order symmetric matrix system are presented. Numerical results are presented in order to illustrate the effectiveness of the proposed method. Extension to structural-acoustic problems are in progress [11] and will be presented in further papers.

\section{ACKNOWLEDGEMENTS}

This research is partially carried out within the 6th Framework Programme of the European Commission (InMAR project) under Grant No. NMP2-CT-2003-501084.

\section{REFERENCES}

1. Göransson P. Acoustic finite element formulation of a flexible porous material—a correction for inertial effects. Journal of Sound and Vibration 1995; 185(4):559-580.

2. Kehr-Candille V, Ohayon R. Elastoacoustic damped vibrations-finite element and modal reduction methods. In New Advances in Computational Structural Mechanics, Ladevèze P, Zienkiewicz OC (eds). Elsevier: Amsterdam, 1992; 321-334.

3. Bermúdez A, Rodríguez R. Modelling and numerical solution of elastoacoustic vibrations with interface damping. International Journal for Numerical Methods in Engineering 1999; 46(10):1763-1779.

4. Davidsson P, Wernberg P-A, Sandberg G. Using the finite element method for control of sound fields in room acoustics. In 15th Nordic Seminar on Computational Mechanics, Lund E, Olhoff N, Stegmann J (eds), 18-19 October 2002, Aalborg, Denmark.

5. Pierce AD. Acoustics: An Introduction to Its Physical Principles and Applications. Acoustical Society of America: New York, 1989.

6. Morand HJ-P, Ohayon R. Fluid-Structure Interaction. Wiley: New York, 1995.

7. Ohayon R, Soize C. Structural Acoustics and Vibration. Academic Press: San Diego, 1998.

8. Ohayon R. Reduced models for fluid-structure interaction problems. International Journal for Numerical Methods in Engineering 2004; 60(1):139-152.

9. Bermúdez A, Durán RG, Rodríguez R, Solomin J. Finite element analysis of a quadratic eigenvalue problem arising in dissipative acoustics. SIAM Journal on Numerical Analysis 2000; 38(1):267-291.

10. Blevins RD. Formulas for Natural Frequency and Mode Shapes. Krieger Publishing Company: Malabar, Florida, 1993.

11. Deü J-F, Larbi W, Ohayon R. Structural-acoustic vibration and transient problems with interface damping. Third M.I.T. Conference on Computational Fluid and Solid Mechanics, Cambridge, U.S.A., 14-17 June 2005. 